\title{
Glucose movement into rat uterine fluid
}

\author{
H. J. Leese, Sue Aldridge and Theresa Kiernan \\ Department of Biology, University of York, York YOI SDD, U.K.
}

\begin{abstract}
Summary. The uterine horns of anaesthetized rats were cannulated and $2.5 \mathrm{ml} 0.9 \%$ $\mathrm{NaCl}$ at $37^{\circ} \mathrm{C}$ were recirculated for up to $90 \mathrm{~min}$. Glucose appeared in the medium, reaching a concentration of approximately $1 \mathrm{mM}$. Various aspects of the transport process were examined, and it is suggested that the glucose is mainly derived from the plasma, and probably enters the uterine lumen by a process involving facilitated diffusion.
\end{abstract}

\section{Introduction}

The composition of the uterine secretions is known for a number of species, but relatively little is known about the way in which they are formed. Most work has been concerned either with uterine fluids collected by ligation over long periods (several days in some species) or has involved the analysis of flushings of the uterine lumen. Glucose is a characteristic component of uterine secretions in a number of species, and was first reported to be present in the rat uterine lumen by Shih, Kennedy \& Huggins (1940). We have therefore examined the appearance of this sugar in saline medium recirculated through the uterine lumen of anaesthetized virgin rats for periods of up to $90 \mathrm{~min}$.

\section{Materials and Methods}

The perfusion circuit was similar to that previously described for the rabbit oviduct (Leese \& Jeffries, 1977). A $2.5 \mathrm{ml}$ volume of medium from a water-jacketed sampling reservoir at $37^{\circ} \mathrm{C}$ was withdrawn by a peristaltic pump (Gilson) at a rate of $100 \mu \mathrm{l} / \mathrm{min}$. The medium was passed through a second heating jacket at $37^{\circ} \mathrm{C}$ before entering the uterine lumen, which was cannulated with nylon tubing (i.d. $1.00 \mathrm{~mm}$, o.d. $1.34 \mathrm{~mm}$; Portex Ltd, Hythe, Kent). The medium was then returned to the reservoir. Vinyl tubing (i.d. $0.63 \mathrm{~mm}$, o.d. $1.4 \mathrm{~mm}$ : Portex Ltd.) was used to connect the various parts of the circuit and the uterus was perfused in the direction cervix to oviduct. The perfusion medium was $0.9 \%(\mathrm{w} / \mathrm{v}) \mathrm{NaCl}$ containing streptomycin $(100 \mu \mathrm{g})$ and penicillin (200 units: both from Sigma Chemical Company Ltd, London). The initial $\mathrm{pH}$ of the medium was around $6 \cdot 0$, reflecting the $\mathrm{pH}$ of the distilled water used to make the saline, but as the perfusion began, the pH rose, reaching 7.0 after $10-15 \mathrm{~min}$ and remaining at this value for the remainder of the experiment. Both uterine horns were routinely perfused by duplicating the perfusion circuit described. Evaporative losses from the circuits were insignificant. The rats $(200-250 \mathrm{~g})$ were anaesthetized with $35 \mathrm{mg}$ pentobarbitone sodium (Sagatal: May \& Baker Ltd, Dagenham)/ $\mathrm{kg}$ body weight and laid on a warm tray to help maintain their body temperature. Serial samples $(50 \mu \mathrm{l})$ of circulating fluid were analysed for glucose by an automated method (GOD-Perid: Boehringer Mannheim GMBH) on a Technicon Autoanalyser. The results were corrected for the decreasing circulating volume and are expressed as total $\mu \mathrm{mol}$ glucose appearing. Vaginal smears were taken with a swab moistened with saline and stained with haematoxylin and eosin. 


\section{Results}

Text-figure 1 shows that glucose appeared steadily in the fluid perfusing the uterine lumen to reach a concentration of about $1 \mathrm{mM}$ after $90 \mathrm{~min}$. No consistent difference in glucose appearance was ever observed between the left and right uterine horns. Throughout the experiments, the plasma glucose was approximately $11 \mathrm{mM}(2 \mathrm{mg} / \mathrm{ml})$. As the standard error bars in Text-fig. 1 indicate, there was considerable variation between experiments, and an attempt was made to see whether this could be accounted for by the stage of the oestrous cycle. Experiments were also carried out on rats 6 days post coitum because implantation in the rat occurs at about this time (Mayer, 1963) and glucose is likely to be of importance in the uterine lumen at this early stage of pregnancy. However, as the data in Table 1 indicate, there were no significant differences between the rates of glucose appearance in the various states examined.

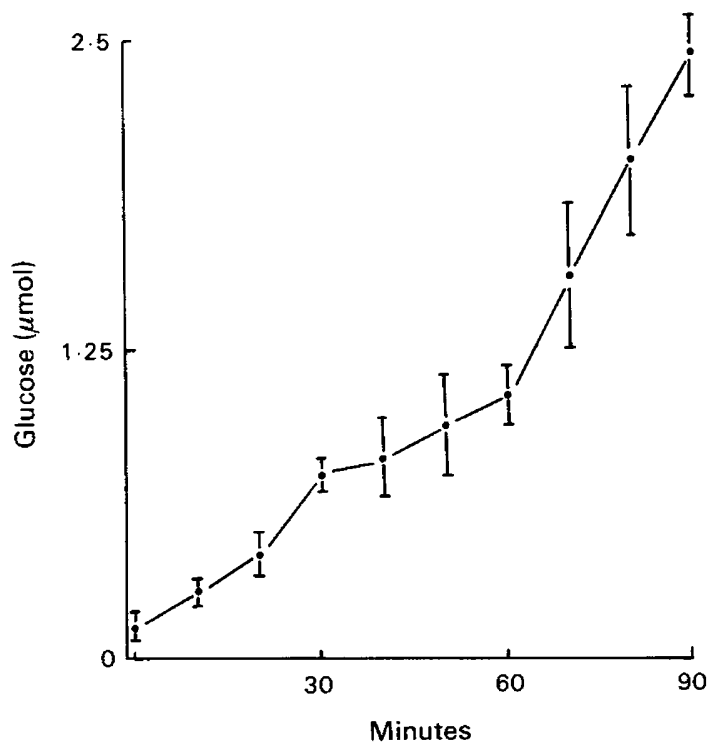

Text-fig. 1. The appearance of glucose in fluid circulating through the rat uterine lumen in vivo. Values are mean \pm s.e.m. of at least 8 uteri. Data from the left and right uterine horns have been pooled.

Table 1. Rates of glucose appearance in fluid circulating through the rat uterine lumen in vivo for $60 \mathrm{~min}$

\begin{tabular}{lc}
\hline Condition of rat & $\begin{array}{c}\text { Rate of glucose appearance } \\
(\mu \mathrm{mol} / \mathrm{h})\end{array}$ \\
\hline Proestrous & $0.44 \pm 0.12(6)$ \\
Oestrous & $0.36 \pm 0.04(7)$ \\
Metoestrous & $0.51 \pm 0.17(7)$ \\
Dioestrous & $0.25 \pm 0.04(6)$ \\
6 days post coitum & $0.44 \pm 0.09(8)$ \\
\hline
\end{tabular}

Values are mean \pm s.e.m. with the number of determinations in parentheses. None of the differences between the means was statistically significant by Student's $t$ test $(P>0.05)$.

Doubling or halving the flow rate of circulating medium had no effect on the rate of glucose appearance in a given uterine horn, suggesting that a flow rate of $100 \mu \mathrm{l} / \mathrm{min}$ was adequate. However, several experiments were carried out in which the circulation of fluid to one uterine 
horn was stopped after $30 \mathrm{~min}$ (with the uterine lumen full of fluid) and then re-started after a further $30 \mathrm{~min}$, continuing up to $90 \mathrm{~min}$. The control horn was perfused continuously. As the data in Text-fig. 2 show, glucose appeared at the same rate during the period when the circulation was stopped, as it did before and after the stoppage, suggesting that merely filling the uterus with fluid was sufficient to ensure optimal rates of glucose appearance.

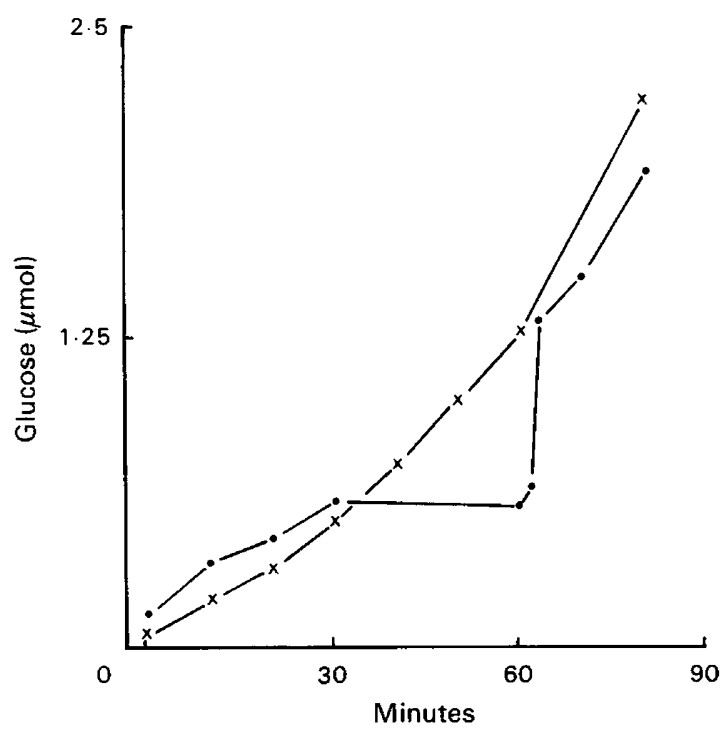

Text-fig. 2. The appearance of glucose in fluid circulating through the left $(O)$ and right $(x)$ uterine horns of a single rat. The circulation of fluid through the left horn was stopped for the period between 30 and 60 $\min$.

To determine whether the glucose was being utilized by the uterus, two experiments were carried out in which $\mathrm{D}-\left[\mathrm{U}-{ }^{14} \mathrm{C}\right]$ glucose and $\mathrm{L}-\left[1-{ }^{14} \mathrm{C}\right]$ glucose were added to the perfusion fluid together with the corresponding unlabelled sugars to a concentration of $1 \mathrm{mM}$. After $1 \mathrm{~h}$ perfusion an average of $82 \%$ and $89 \%$ of the radioactivity derived from the $\mathrm{D}^{-}$and L-glucose, respectively, remained in the fluid, suggesting that relatively little consumption or reverse flux of glucose had taken place.

In animals killed before the start of perfusion there was virtually no glucose output, suggesting that an intact blood cirulation was essential for detection of a glucose secretion. In animals killed after $30 \mathrm{~min}$ there was either a levelling off in glucose appearance, or occasionally a glucose disappearance, such that all of the glucose which had appeared after $30 \mathrm{~min}$ had disappeared by $90 \mathrm{~min}$. When the luminal fluid was pre-loaded with glucose at a concentration of $11 \mathrm{~mm}$ (i.e. similar to that in the plasma) there was no detectable increase or decrease in glucose concentration, suggesting that the rat uterus was unable to bring about the overall uphill or active transport of glucose.

In view of this, attempts were made to determine whether the glucose transport was by passive or facilitated diffusion. Phloretin, which is an inhibitor of the facilitated transport of glucose in red cells and other systems (Le Fevre \& Marshall, 1959), was added to the luminal fluid at a concentration of $10^{-4} \mathrm{M}$ after $30 \mathrm{~min}$, but had no significant effect on the rate of glucose appearance. Sugars which share the same transport system as glucose can influence glucose fluxes, either by competition or by inducing the phenomenon of countertransport (Le Fevre, 1975). However, neither galactose nor 3-O-methyl-D-glucose added to the lumen at a concentration of $10 \mathrm{~mm}$ after $30 \mathrm{~min}$ had a significant effect on the rate of glucose apperance. 


\section{Discussion}

The present experiments measured the net rate of glucose appearance in the uterine lumen, i.e. the difference between the glucose flux in a luminal direction and the glucose flux in the opposite direction, and the results show that a glucose movement into the rat uterine lumen may readily be observed over relatively short periods in vivo.

While Heap (1962) found significant differences in the amounts of carbohydrate flushed from the uterine horns of rats at oestrus and dioestrus, we were unable to detect significant differences in the rate of glucose appearance at various stages of the oestrous cycle, or at 6 days after mating. We consider that variation between the animals was probably masking any effect and that this difficulty might be circumvented by measuring the rates of glucose movement across an in-vitro uterine preparation for which the number of variables is less than those encountered in vivo.

The experiments on dead animals indicated the plasma as the probable source of the glucose appearing in the uterine lumen. However it is possible that the glycogen present in the cells of the endometrium could be contributing to the glucose appearance, either by being broken down in the endometrium and then being secreted, or by being secreted as an intact macromolecule to be broken down in the lumen.

Although the appreciable rate of glucose appearance observed would suggest that its movement is mediated in some way, it is not possible from the present data to be sure of the precise transport mechanism. However, Walters (1976) has reported hormone-sensitive carrier mechanisms for sugars and amino acids in the rat uterus and these would favour some facilitated diffusion process. In our hands the inhibitor phloretin was ineffective and the attempts to induce competition or counterflow effects were unsuccessful. It is possible that the membrane of the endometrial cells which borders the lumen is insensitive to the action of phloretin and that this inhibitor and other sugars tested would be more effective if added to the contraluminal membranes of the cell. This is difficult to accomplish in vivo and suggests that further progress in elucidating the mechanisms underlying the glucose secretion is more likely to come from using an in-vitro uterine preparation such as that developed by Levin \& Edwards (1968).

We are grateful to the Medical Research Council for a research grant.

\section{References}

Heap, R.B. (1962) Some chemical constituents of uterine washings: a method of analysis with results from various species. J. Endocr. 24, 367-378.

Leese, H.J. \& Jeffries, K.S. (1977) Evidence for the facilitated diffusion of glucose into rabbit oviductal fluid. J. Reprod. Fert. 51, 93-97.

Le Fevre, P.G. (1975) The present state of the carrier hypothesis. Current Topics in Membranes and Transport 7, 109-215.

Le Fevre, P.G. \& Marshall, J.K. (1959) The attachment of phloretin and analogues to human erythrocytes in connection with inhibition of sugar transport. J. biol. Chem. 234, 3022-3026.
Levin, R. J. \& Edwards, F. (1968) The transuterine endometrial potential difference, its variation during the oestrous cycle and its relation to uterine secretions. Life Sci. 7, 1019-1036.

Mayer, G. (1963) Delayed nidation in rats: a method of exploring the mechanisms of ovo-implantation. In Delayed Implantation, pp. 213-231. Ed. A. C. Enders. University of Chicago Press.

Shih, H.E., Kennedy, J. \& Huggins, C. (1940) Chemical composition of uterine secretions. Am. J. Physiol. 130, 287-291.

Walters, E.M.R. (1976) Amino acid and sugar transport mechanisms in the rat uterine luminal epithelium. Diss. Abstr. 36, 5457-5458B.

Received 1 December 1978 